\title{
Stretchable Hybrid Bilayered Luminescent Composite Based on the Combination of Strain-Induced and Triboelectrification-Induced Electroluminescence
}

\author{
Yanghui Chen, ${ }^{\dagger,+, \S}$ Xiaoyan Wei, ${ }^{\dagger,+}$ Huayang Li, ${ }^{\dagger,+}$ Youjun Fan, ${ }^{\dagger,+}$ Weiguo $\mathrm{Hu}^{\dagger, \text {, }}$ \\ and Guang Zhu, ${ }^{*},+, \| \odot$ \\ ${ }^{\dagger}$ CAS Center for Excellence in Nanoscience, Beijing Key Laboratory of Micro-Nano Energy and Sensor, Beijing Institute of \\ Nanoenergy and Nanosystems, and ${ }^{\S}$ Institute of Semiconductors, Chinese Academy of Sciences, Beijing 100083, China \\ ${ }^{\ddagger}$ School of Nanoscience and Technology, University of Chinese Academy of Sciences, Beijing 100049, China \\ "New Materials Institute, Department of Mechanical, Materials and Manufacturing Engineering, University of Nottingham Ningbo \\ China, Ningbo 315100, China
}

\section{Supporting Information}

ABSTRACT: High luminescence intensity from materials that are excited by external stimuli is highly desired. In this work, a stretchable hybrid luminescent composite (HLC) that has multiple luminescence modes is reported. The luminescence can be excited either by externally applied mechanical strain or by a moving object that slides against the HLC. When the HLC is deformed, such as being twisted or folded, the $\mathrm{ZnS} / \mathrm{Cu}$ phosphor experiences mechanical strain that trigger the mechanoluminescence (ML) of the phosphors. Moreover, as the HLC slides against a contact object, the triboelectrification at the contact interface induces the electroluminescence of phosphor. Here, a series of internal and external factors were studied on how they influence the luminescent intensity. It is found that the luminescent intensity from the

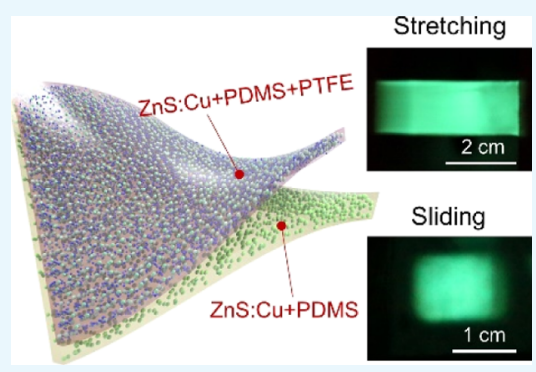
two modes can be superposed. The HLC material was used to fabricate a fiber-based luminescent device that can be driven by air flow. The overall luminescent intensity is enhanced by over $72 \%$ compared to that obtained solely from the ML. The HLC reported in this work has such potential applications as self-powered light sources and sensors as means of detecting dynamic motions and interaction.

\section{INTRODUCTION}

Methods for converting mechanical energy into light emission are promising for use in illumination, ${ }^{1}$ displays, ${ }^{2}$ and stress sensors. ${ }^{3,4}$ Conventionally, strain-induced luminescence, which is also known as mechanoluminescence $(\mathrm{ML})$, is the most common method for achieving such energy conversion. ${ }^{5,6}$ For example, Jha and Chandra reported the impulsive excitation of the ML in $\mathrm{SrAl}_{2} \mathrm{O}_{4}: \mathrm{Eu}^{2+}$ and $\mathrm{Dy}^{3+}$ phosphors. ${ }^{7}$ However, this mechanism has several limitations that affect its practical applications. First, the high-intensity ML materials are usually inorganic materials, ${ }^{8}$ such as quartz, ${ }^{9}$ rare-earth ion-doped aluminates, ${ }^{10}$ and doped zinc sulfide. ${ }^{11}$ To generate ML, their large Young's modulus results in a high threshold pressure at the scale of several $\mathrm{MPa}{ }^{12,13}$ Second, the ML is usually accompanied by the material damage and the decay of the luminescence intensity, which affect ML's reproducibility. ${ }^{14,15}$ Recently, novel triboelectrification-induced luminescence (TIEL) that could convert kinetic energy into light emission was reported. ${ }^{16}$ The TIEL exhibited an exceptionally low threshold that was 2-3 orders of magnitude lower than the conventional ML. It also possessed high luminescence intensity. If the TIEL and the ML could be achieved in a single material, significant luminescence intensity from the mechanical-optical conversion can be achieved.
In this work, we report a stretchable hybrid luminescent composite (HLC) that could generate both ML and TIEL under different mechanical stimuli. The HLC possessed two luminescence modes. The luminescence could be excited either by externally applied mechanical strain or by contacting objects that interact with the HLC. On the one hand, when it was deformed, such as being twisted or folded, the $\mathrm{ZnS} / \mathrm{Cu}$ phosphors experienced mechanical strain that triggered the $\mathrm{ML}$ of the phosphors. On the other hand, as the multilayered composite luminescent material contacted with an external moving object, the triboelectrification at the contact interface induced electroluminescence of the phosphors. A series of internal and external parameters were investigated on how they influenced luminescent intensity for each of the modes above. It was found that the luminescent intensity from the two modes could be superposed. We used the HLC material to fabricate a fiber-based light source that could be driven by air flow. The overall luminescent intensity was enhanced by over $72 \%$ compared to that obtained solely from the ML. The HLC reported in this work has such potential applications as self-

Received: June 11, 2019

Accepted: September 10, 2019

Published: November 25, 2019 
(a)

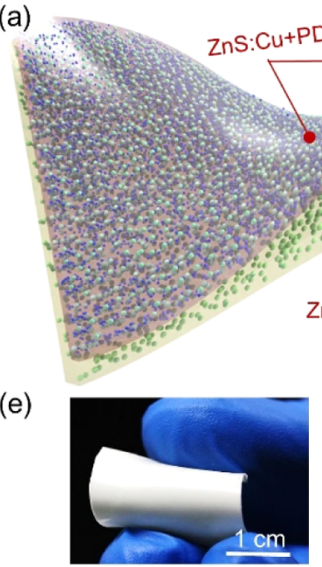

(f)

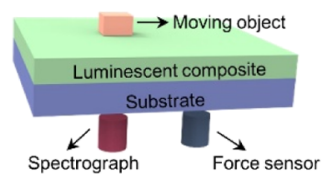

(b)

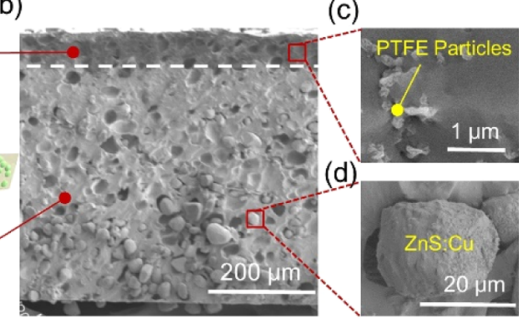

(1)

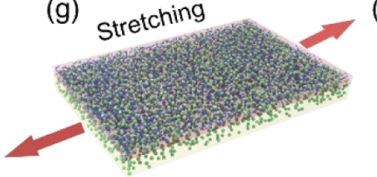

(h)

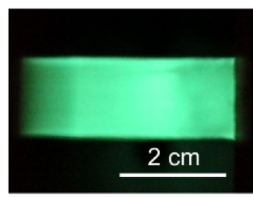

(i)

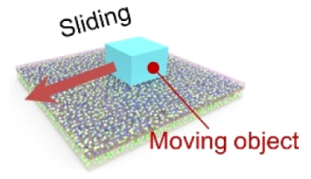

(j)

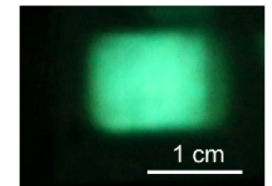

Figure 1. Structure of the stretchable HLC. (a) Schematic of the HLC. (b) Cross-sectional SEM image of the HLC. (c) Magnified image of the PTFE nanoparticles distributed in the PDMS matrix. (d) Magnified image of phosphor. (e) Photograph of an as-fabricated HLC. (f) Diagram of the experimental setup used for the optical measurement. Schematic illustration of the external stimuli from (g) stretching and (i) sliding with a contact object. Photographs of the ML (h) and the TIEL (j) from the HLC.
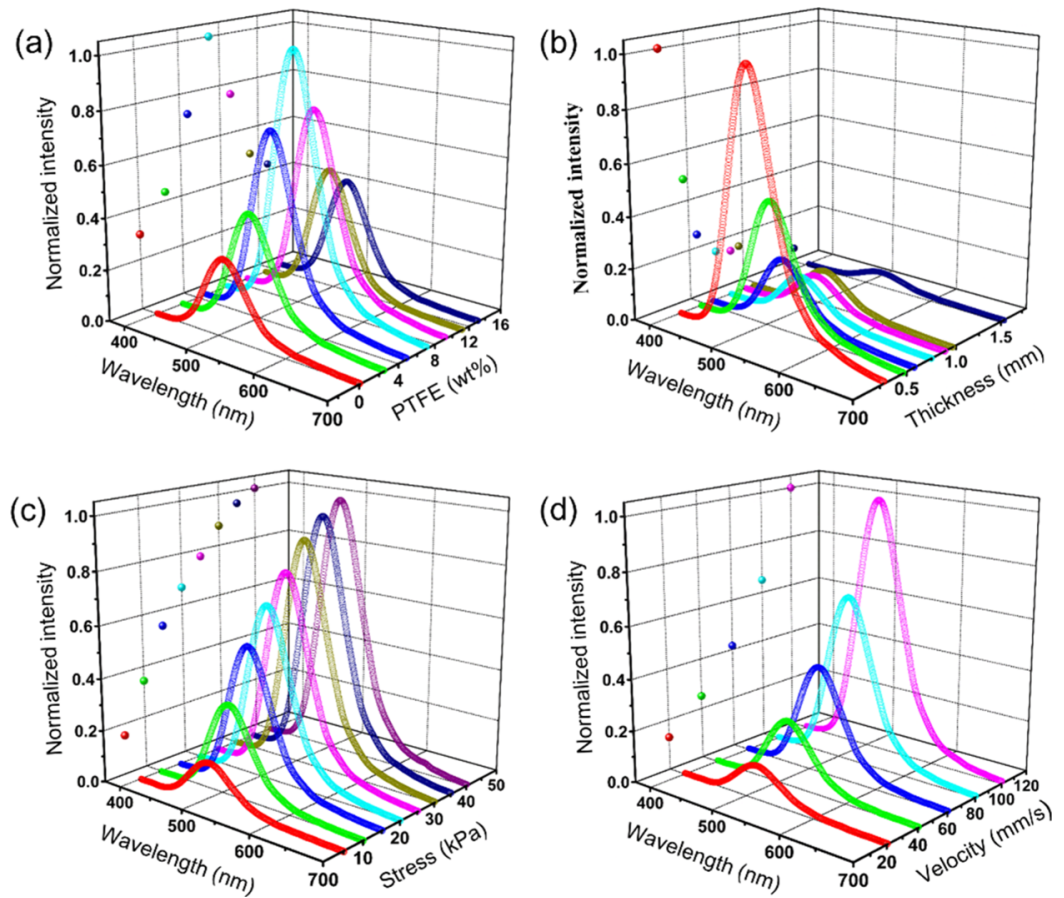

Figure 2. Optical measurement results of the TIEL from the HLC. (a) Luminescence spectra with increasing contents of the PTFE nanoparticle. (b) Luminescence spectra with increasing the HLC thickness. (c) Luminescence spectra as the stress at the contact surfaces varies from 5 to 40 $\mathrm{kPa}$. (d) Luminescence spectra as the velocity of the sliding object varies.

powered light sources and sensors as means of detecting dynamic motions and interaction.

\section{RESULTS AND DISCUSSION}

The schematic diagram of the HLC is shown in Figure 1a. The HLC basically consisted of a phosphor and a polydimethylsiloxane (PDMS) matrix; and polytetrafluoroethylene (PTFE) nanoparticles were added into the top layer of the HLC. The cross-sectional scanning electron microscope (SEM) view of the HLC is presented in Figure 1b, which clearly shows a two- layered structure. The $\mathrm{ZnS} / \mathrm{Cu}$ phosphor was well dispersed in the PDMS matrix that was used for transferring externally applied stress to the particles. The magnified view in Figure 1c presents the distribution of the PTFE nanoparticles within the HLC. These particles play two roles. In addition to reducing the friction coefficient between the HLC and a contact object, they also enhanced the triboelectrification between the contact surfaces because the PTFE strongly attracts negative triboelectric charges. ${ }^{17,18}$ The magnified SEM image of the phosphor is shown in Figure $1 \mathrm{~d}$. The $\mathrm{ZnS} / \mathrm{Cu}$ phosphor has 


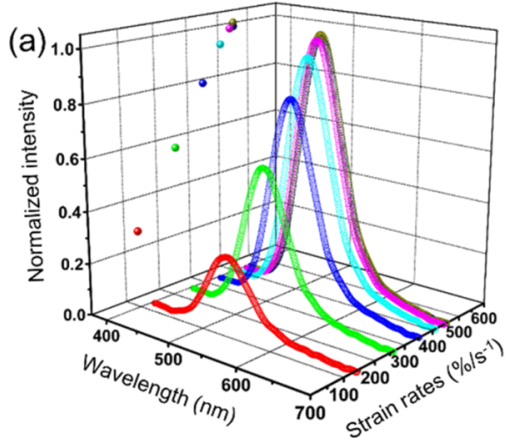

(b)
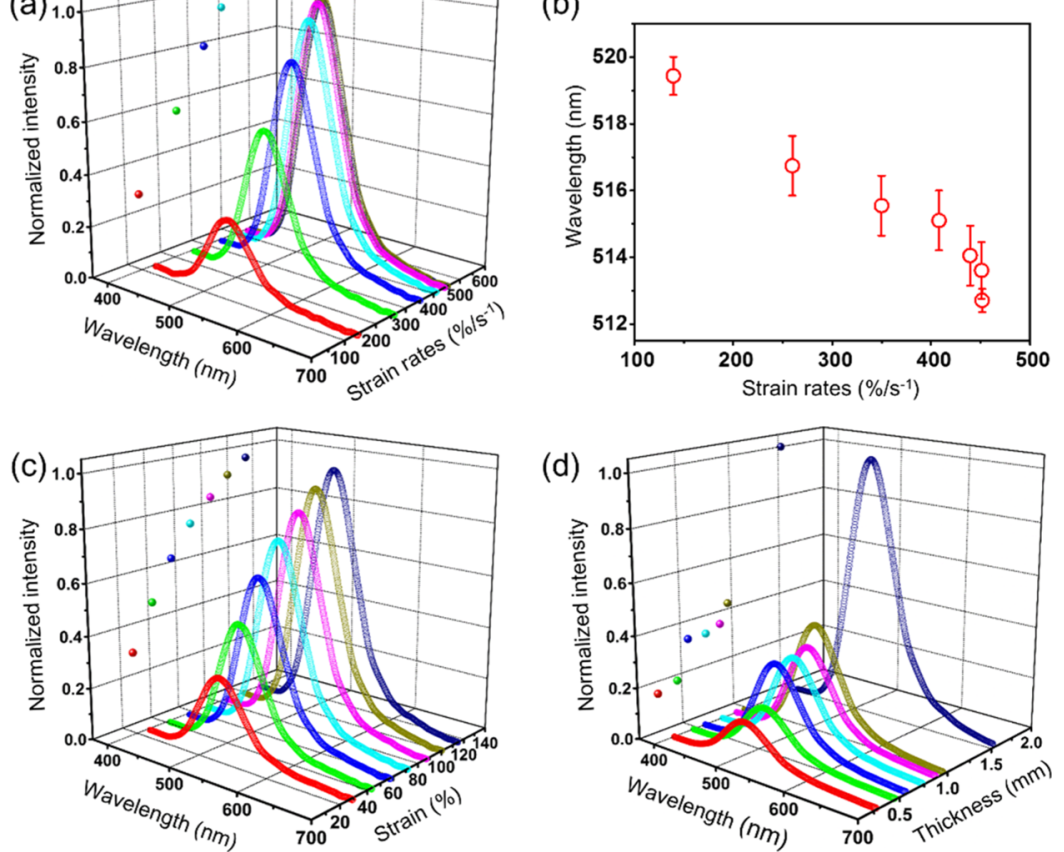

Figure 3. Optical characteristics of the ML from the HLC. (a) ML spectra and (b) peak wavelength with increasing the externally applied strain rate. (c) ML spectra under different strains of the HLC. (d) Dependence of the ML spectra on the thickness of the HLC.

a diameter of $\sim 26 \mu \mathrm{m}$. It is a commonly used material that could be excited by either mechanical stress ${ }^{19-22}$ or a charging electric field. ${ }^{23-25}$ The X-ray diffraction (XRD) patterns show that the phosphors have a wurtzite structure (Figure S1). A photograph of the as-fabricated HLC is shown in Figure 1e. A diagram of the experimental setup used for spectral measurement is presented in Figure 1f. An optical fiber-based probe collected the optical emission from the back of the HLC. The detailed fabrication process is discussed in Methods.

The HLC has two basic luminescence modes for the mechanical-optical conversion, as illustrated in Figure $1 \mathrm{~g}-\mathrm{j}$. The first mode is based on the ML. The luminescence can be induced by applying a mechanical force to the HLC. When stretched, the $\mathrm{ZnS} / \mathrm{Cu}$ phosphor produces $\mathrm{ML}$ (Video $\mathrm{S} 1$ ). The fundamental mechanism of the ML process has been extensively studied in the literature. ${ }^{26,27}$ Basically, the straininduced electric field of the charged dislocations causes band bending of the $\mathrm{ZnS}$ crystal. As a result, the electrons trapped in the shallow donor level tunnel to the conduction band. When the excited electrons of the $\mathrm{Cu}^{2+}$ ions fall back to the $t_{2}$ level of the $\mathrm{Cu}^{2+}$, photons are emitted (Figure S2). ${ }^{26}$ A photograph of the $\mathrm{ML}$ as the HLC was being stretched is presented in Figure 1 h. For the second mode, if a contact object slides on the top surface of the HLC, the phosphor underneath the sliding trajectory can also produce luminescence [Figure 1i,j, Video S2]. This type of luminescence is referred to as TIEL, as previously reported. ${ }^{16}$ Essentially, the TIEL relies on the coupling of triboelectrification and electroluminescence. When a relative sliding occurs between two dissimilar materials, surface charge transfer takes place due to the triboelectrification effect. The surface triboelectric charges generate a transient electric field along the sliding trajectory, exciting the phosphor underneath. As indicated by the discussion above, the HLC can produce light emission when triggered by multiple types of stimuli.
In the following sections, the two luminescence modes are investigated in detail, especially on respective influencing factors. First, the major factors that influence the TIEL are investigated in Figure 2. On the one hand, the two parameters regarding the design of the material are shown in Figure 2a,b, that is, the PTFE concentration and the overall thickness, respectively. As the content of the PTFE nanoparticles increased, the luminescence intensity initially increased and then decreased, resulting in an optimal weight percentage at $7.0 \%$. The luminescent intensity was enhanced by over $300 \%$ compared to that obtained from the luminescent composite without PTFE nanoparticles. This finding shows that the addition of the PTFE nanoparticles do promote the triboelectrification between the two contacting surfaces. However, it was found that excessive PTFE nanoparticles reduced the transparency of the HLC, which blocked the luminescence from transmitting through the HLC. We also fabricated onelayered HLC and bilayered HLC. The overall thickness of the one and two layer structure is the same, which is about 0.33 $\mathrm{mm}$. The content of PTFE nanoparticles in the one-layered HLC and the first layer of bilayered HLC was $6.98 \mathrm{wt} \%$. There were no PTFE particles in the second layer of the bilayered HLC. The TIEL of the one-layered HLC and bilayered HLC was measured at a velocity of $10 \mathrm{~mm} / \mathrm{s}$ and a stress of $25 \mathrm{kPa}$, as shown in Figure S3. The TIEL intensity of the bilayered HLC is over twice that of the one-layered HLC. Soon, Jeong et al. reported one-layered $\mathrm{ML}$ devices. ${ }^{20,28}$ Wang et al. reported a multilayered ML device using the polyvinylidene fluoride film to enhance ML intensity. ${ }^{29}$ Wei et al. reported a multilayered TIEL device using $\mathrm{ZnS} / \mathrm{Cu}$ particles as a luminescent layer and fluorinated ethylene propylene (FEP) film as an electrification layer. ${ }^{16}$ However, the device is not stretchable. The advantage of the bilayered HLC is that the bilayered HLC can integrate ML and TIEL. The bilayered HLC also show higher luminescence intensity than the one-layered HLC when they were driven by the same mechanical stimulus. In addition, the 
(a)

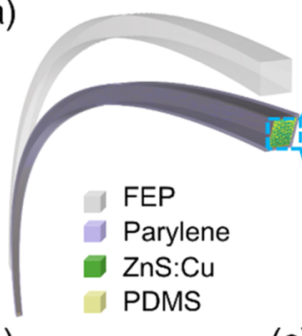

(b)
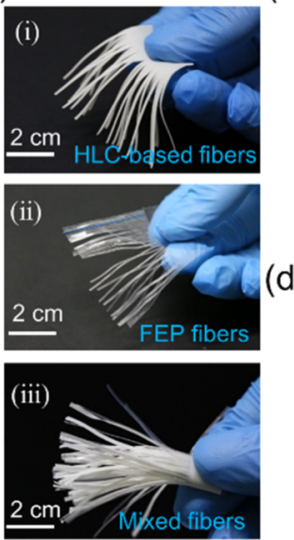

(c)
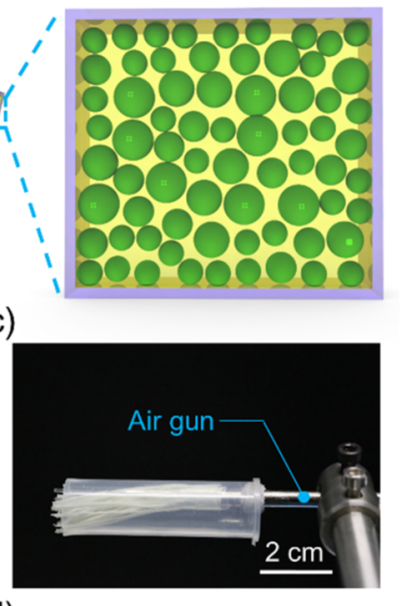

(e)
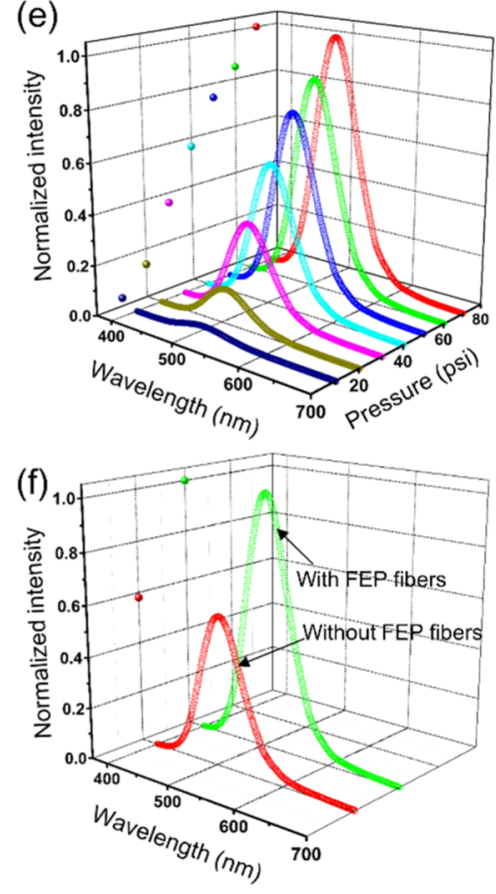

Figure 4. Demonstration of the HLC used in an air-driven luminescence device. (a) Schematic diagram of the core-shell-structured HLC fibers, with a magnified view of the core-shell structure. (b) Photographs of the HLC-based fibers, FEP fibers, and mixed fibers with an average width of $\sim 1 \mathrm{~mm}$. (c) Static image of the wind-driven luminescence device. (d) Photograph of the wind-driven device with flowing air. (e) Dependence of the luminescence spectra on the releasing pressure of the air gun. (f) Luminescence spectra obtained from the mixed fibers and the HLC-based fibers, respectively.

overall thickness of the HLC could also affect the luminescence intensity with constant thickness of the first layer. As the thickness increased from 0.28 to $1.53 \mathrm{~mm}$, the luminescence intensity decreased substantially by over $90 \%$ of the original value. This can be intuitively explained by the fact that increasing the thickness prevents the emitted light from escaping through the HLC. On the other hand, another two parameters regarding how the contact object interacted with the HLC were also examined, as shown in Figure $2 \mathrm{c}, \mathrm{d}$. The luminescence intensity was found to be very sensitive to the applied pressure at the contact interface (Figure 2c). Indeed, as the pressure increased from 5 to $40 \mathrm{kPa}$ (velocity, $80 \mathrm{~mm} / \mathrm{s}$ ), the luminescence intensity was enhanced by a factor of 6.5 . This enhancement is attributed to the fact that high contact pressure favors the generation of high-density triboelectric charges. ${ }^{30}$ As a result, a high electric field can be obtained to excite the luminescence. As the pressure surpassed $40 \mathrm{kPa}$, the luminescence intensity tended to saturate because the triboelectric charges density reached a maximum limit (Figure S4). ${ }^{31}$ Besides, as shown in Figure $2 \mathrm{~d}$, the velocity of the sliding object exerted a profound effect on the luminescence intensity (stress, $20 \mathrm{kPa}$ ). Similar observation was also reported in previous research. ${ }^{16}$ Higher sliding velocity essentially increased the equivalent frequency of the changing electric field, ${ }^{32}$ which benefited the electroluminescence of the phosphor.

Second, a number of factors influencing the $\mathrm{ML}$ were investigated with experimental results shown in Figure 3. A linear motor was adopted to apply a periodic stretching strain to the HLC. The strain rate substantially influenced the luminescence intensity. The ML intensity had a positive linear correlation with the strain rate, as shown in Figure 3a. This observation was consistent with previous reports. ${ }^{20}$ When the average strain rate increased from 139 to $451 \% / \mathrm{s}$, the ML intensity increases by approximately $377 \%$ (the initial length of the HLC is $7 \mathrm{~mm}$ ). Detailed observations on the peak wavelength revealed that the ML spectrum shifted slightly to shorter wavelength as the strain rate increased. This is attributed to the activation of different energy levels of the $\mathrm{ZnS} / \mathrm{Cu}$ phosphor, as reported previously. ${ }^{28}$ Shown in Figure $3 \mathrm{~b}$, the peak wavelength shifted from 519 to $513 \mathrm{~nm}$. Besides, the applied strain was also positively correlated with the luminescence intensity, as exhibited in Figure 3c. The luminescence intensity increased by about 3.5 times when the strain increased from 28.6 to $128.6 \%$. The maximum principal stress is proportional to the strain of the film. ${ }^{33}$ In addition, larger HLC thicknesses resulted in higher luminescence intensities. The luminescent intensity increased by about 5.6 times when the thickness of the HLC changed from 0.28 to $1.53 \mathrm{~mm}$, as shown in Figure 3d. This is because that more phosphors were involved in the ML process when the thickness of the HLC increased. Then, more generated emissions could be obtained during the same detection time of the spectrometer.

Based on the two luminescence modes discussed above, a wind-driven luminescent device was fabricated, in which the TIEL and the ML could be superimposed to promote the overall luminescence intensity. We fabricated two kinds of HLC with and without embedded PTFE particles on the surface. The HLC were sliced into fibers with a width of $\sim 1$ $\mathrm{mm}$ and a length of $3.5 \mathrm{~cm}$. The fibers were rolled up around a gas tube at one end, leaving the other end of the fibers freestanding. There is no significant difference in the luminous intensity between two kinds of HLCs, as shown in Figure S5. The devices showed little TIEL. It is because PTFE and PDMS are very close in the triboelectric series. ${ }^{34}$ There were little 
transferred surface triboelectric charges between the layer with PTFE particles and the layer without PTFE particles. Then, we fabricated two types of fibers. As illustrated in Figure $4 \mathrm{a}$, the device was composed of two types of fibers bundled together. The first type had a core-shell structure. The core was based on the HLC, and the shell was made of parylene coating with a thickness of $2 \mu \mathrm{m}$ [Figure $4 \mathrm{~b}(\mathrm{i})$ ]. This shell material not only served as a protective coating that promoted the mechanical robustness of the HLC-based fiber against external stimuli but also acted as an electrification material that generated and retained the triboelectric charges. Another type of the fibers was made of plain FEP, as shown in Figure $4 \mathrm{~b}$ (ii). FEP is transparent and then will not prevent the emitted light from being transmitted to the spectrometer. It is a good triboelectrically negative material. ${ }^{35}$ Much surface triboelectric charges could be generated on the parylene-coated HLC, which could generate TIEL. The device was fabricated through assembling the two types of fibers together at one end and leaving the other end of the fibers free-standing. The photograph of the mixed fibers is shown in Figure $4 \mathrm{~b}$ (iii). To trigger the luminescent device, air flow was introduced from the assembled end along the length of the fibers, as revealed in Figure 4c. Excited by the air flow, the mixed fibers fluttered violently, producing high-intensity luminescence, as shown in Figure 4d. The luminescence comes from two sources. First, the strain incurred within the HLC-based fibers generates $\mathrm{ML}$ of the phosphor. Second, the contact between the FEP fibers and the HLC-based fibers induced the TIEL. As the air flow pressure increased, the luminescence intensity became significantly enhanced (Figure 4e). This observation was supported by the experimental data presented in Figure $4 \mathrm{f}$. If the FEP fibers were removed, the HLC-based fibers alone produced a luminescence intensity that was $42 \%$ lower than that produced by the hybrid fibers. This is because that the HLC-based fibers mainly generated the ML with few TIEL.

\section{CONCLUSIONS}

In summary, in this work, a stretchable bilayered luminescent composite is developed based on combination of straininduced luminescence and TIEL. The composite film embedded with green light-emitting doped $\mathrm{ZnS}$ particles exhibits two luminescence modes. The luminescence can be excited either by externally applied mechanical strain or by contacting objects that interact with the HLC. The surface triboelectric charges resulting from the sliding motion generate a charging electric field along the sliding trajectory, thereby exciting the underlying phosphors. The low-pressure threshold $(5 \mathrm{kPa})$ of the electroluminescence implies that these devices have wide applications. Furthermore, a gas flow system was presented to simulate the effect of wind, and increasing the air compressor discharge pressure was found to increase the luminescence of the composite film. The luminescent intensity collected from the device decreased after elimination the TIEL of the device. This study demonstrates the potential applications of this HLC in self-powered light sources.

\section{METHODS}

Fabrication of the Stretchable HLC. The stretchable HLC studied here had a two-layered structure. In the first layer, $\mathrm{ZnS} / \mathrm{Cu}$ particles (Shandong Wanji, M-G-AAA) were mixed with the mixture of PDMS and curing agent (10:1 weight ratio, Dow Corning 184) at a weight ratio of 2:1. The mixed solution was spread over an acrylic plate covered with scotch tape and smoothed with a plastic card. After curing at $80{ }^{\circ} \mathrm{C}$ for $1 \mathrm{~h}$, the second layer was spun onto the mixture. The spun speed for the prepared second layer was $2000 \mathrm{rpm}$ (SETCAS Electronics, KW-4B). This second layer consisted of $\mathrm{ZnS} / \mathrm{Cu}$ particles, PDMS matrix, and PTFE nanoparticles (DuPont). The $\mathrm{ZnS} / \mathrm{Cu}$ particles and the PDMS matrix were mixed at a weight ratio of $1: 1$. The ratio between $\mathrm{ZnS} / \mathrm{Cu}$ and PTFE particles changed from 10:0 to 10:3.

Fabrication of the Wind-Driven Luminescent Device. The wind-driven luminescent device consisted of the FEP fibers and the HLC-based composite fibers. An FEP film of $100 \mu \mathrm{m}$ thickness was cut into $5 \mathrm{~cm} \times 3.5 \mathrm{~cm}$ pieces. The FEP fibers were obtained from partially slicing a FEP piece in which each slice had a width of $\sim 1 \mathrm{~mm}$ and a length of $3.5 \mathrm{~cm}$. The FEP piece was cut into fibers by laser (Universal VLS 2.3). $\mathrm{ZnS} / \mathrm{Cu}$ particles were mixed at a weight ratio of $2: 1$ with the PDMS matrix. After curing at $80{ }^{\circ} \mathrm{C}$ for $1 \mathrm{~h}$, the composite material was cut into $5 \mathrm{~cm} \times 3.5 \mathrm{~cm}$ pieces. To obtain the mixed fibers, the HLC was partially sliced the same as the FEP fibers. Then, the HLC-based fibers were deposited with a layer of parylene-N coating by vacuum vapor deposition (PDS-2010 Specialty Coating Systems), forming a core-shell structure. The weight of parylene- $\mathrm{N}$ used in depositing was $2 \mathrm{~g}$. The two types of fibers were bundled together and rolled up around a gas tube that had an external diameter of $5 \mathrm{~mm}$ at one end, leaving the other end of the fibers free-standing.

Optical Characterization. TIEL measurement: a sponge (3M 9448A) was cut into pieces with a size of $0.4 \mathrm{~cm} \times 0.5$ $\mathrm{cm}$. A piece of the sponge covered with a layer of nitrile rubber was fixed on a linear motor (Zolix LMA-TR-200-E10). The covered sponge slid on the HLC via a linear motor. The applied forces between the moving object and the HLC were measured by a dynamometer (Mark-10 M5-20). ML measurement: the HLC was stretched and then released by a linear motor. A piece of the HLC (7 mm length) was used to measure the ML spectra of the HLC at different strain rates. The luminescence spectra of the HLC samples were collected by a spectrometer (Idea Optics NOVA). The spectrometer probe was located at a constant position. The ML and TIEL images were captured by a digital camera (Canon EOS 5D Mark III).

\section{ASSOCIATED CONTENT}

\section{Supporting Information}

The Supporting Information is available free of charge at https://pubs.acs.org/doi/10.1021/acsomega.9b01717.

ML produced by stretching (MP4)

TIEL produced by finger sliding with nitrile gloves (MP4)

XRD pattern of phosphor, showing a wurtzite structure; band diagram of the $\mathrm{ZnS} / \mathrm{Cu}$ phosphors; TIEL of the one-layered HLC and bilayered HLC; luminescence spectra as the stress at the contact surfaces varies from 5 to $50 \mathrm{kPa}$; luminous intensity of the HLC with and without embedded PTFE particles in the surface under air flow (PDF)

\section{AUTHOR INFORMATION}

\section{Corresponding Author}

*E-mail: zhuguang@binn.cas.cn. 


\section{ORCID $\odot$}

Weiguo Hu: 0000-0002-8614-0359

Guang Zhu: 0000-0003-2350-0369

\section{Notes}

The authors declare no competing financial interest.

\section{ACKNOWLEDGMENTS}

This research was supported by the National Key R \& D Project from Ministry of Science and Technology, China (grant nos. 2016YFA0202701 and 2016YFA0202703), National Science Foundation of China (grant no. 51572030), and Natural Science Foundation of Beijing Municipality (grant no. 2162047).

\section{REFERENCES}

(1) Terasaki, N.; Yamada, H.; Xu, C.-N. Ultrasonic wave induced mechanoluminescence and its application for photocatalysis as ubiquitous light source. Catal. Today 2013, 201, 203.

(2) Xu, C.-N.; Zheng, X.-G.; Akiyama, M.; Nonaka, K.; Watanabe, T. Dynamic visualization of stress distribution by mechanoluminescence image. Appl. Phys. Lett. 2000, 76, 179.

(3) Yun, G. J.; Rahimi, M. R.; Gandomi, A. H.; Lim, G.-C.; Choi, J.$\mathrm{S}$. Stress sensing performance using mechanoluminescence of $\mathrm{SrAl}_{2} \mathrm{O}_{4}$ (SAOE) and $\mathrm{SrAl}_{2} \mathrm{O}_{4}: \mathrm{Eu}$, Dy (SAOED) under mechanical loadings. Smart Mater. Struct. 2013, 22, 055006.

(4) Wang, X.; Xu, C.-N.; Yamada, H.; Nishikubo, K.; Zheng, X.-G. Electro-Mechano-Optical Conversions in $\mathrm{Pr}^{3+}$-Doped $\mathrm{BaTiO}_{3-}$ $\mathrm{CaTiO}_{3}$ Ceramics. Adv. Mater. 2005, 17, 1254.

(5) Atari, N. A. Piezoluminescence phenomenon. Phys. Lett. A 1982, $90,93$.

(6) Olawale, D. O.; Dickens, T.; Sullivan, W. G.; Okoli, O. I.; Sobanjo, J. O.; Wang, B. Progress in triboluminescence-based smart optical sensor system. J. Lumin. 2011, 131, 1407.

(7) Jha, P.; Chandra, B. P. Impulsive excitation of mechanoluminescence in $\mathrm{SrAl}_{2} \mathrm{O}_{4}: \mathrm{Eu}^{2+}, \mathrm{Dy}^{3+}$ phosphors prepared by solid state reaction technique in reduction atmosphere. J. Lumin. 2013, 143, 280.

(8) Jha, P.; Chandra, B. P. Survey of the literature on mechanoluminescence from 1605 to 2013. Luminescence 2014, 29, 977.

(9) Reynolds, G. T. Piezoluminescence from a ferroelectric polymer and quartz. J. Lumin. 1997, 75, 295.

(10) Xu, C.-N.; Zheng, X.-G.; Akiyama, M.; Nonaka, K.; Watanabe, T. Dynamic Visualization of Stress Distribution by Mechanoluminescence Image. Appl. Phys. Lett. 2000, 76, 179-181.

(11) Chandra, V. K.; Chandra, B. P.; Jha, P. Strong luminescence induced by elastic deformation of piezoelectric crystals. Appl. Phys. Lett. 2013, 102, 241105.

(12) Jia, Y.; Yei, M.; Jia, W. Stress-induced mechanoluminescence in $\mathrm{SrAl}_{2} \mathrm{O}_{4}: \mathrm{Eu}^{2+}, \mathrm{Dy}^{3+}$. Opt. Mater. 2006, 28, 974.

(13) Chandra, V. K.; Chandra, B. P.; Jha, P. Mechanoluminescence of $\mathrm{ZnS}: \mathrm{Mn}$ phosphors excited by hydrostatic pressure steps and pressure pulses. Phys. B 2014, 452, 23.

(14) Matsui, H.; Xu, C.-N.; Tateyama, H. Stress-stimulated luminescence from $\mathrm{ZnAl}_{2} \mathrm{O}_{4}$ :Mn. Appl. Phys. Lett. 2001, 78, 1068.

(15) Camara, C. G.; Escobar, J. V.; Hird, J. R.; Putterman, S. J. Correlation between nanosecond X-ray flashes and stick-slip friction in peeling tape. Nature 2008, 455, 1089.

(16) Wei, X. Y.; Wang, X.; Kuang, S. Y.; Su, L.; Li, H. Y.; Wang, Y.; Pan, C.; Wang, Z. L.; Zhu, G. Dynamic Triboelectrification-Induced Electroluminescence and its Use in Visualized Sensing. Adv. Mater. 2016, 28, 6656.

(17) Wang, Z. L. Triboelectric Nanogenerators as New Energy Technology for Self-Powered Systems and as Active Mechanical and Chemical Sensors. ACS Nano 2013, 7, 9533.

(18) Zhang, C.; Tang, W.; Han, C.; Fan, F.; Wang, Z. L. Theoretical comparison, equivalent transformation, and conjunction operations of electromagnetic induction generator and triboelectric nanogenerator for harvesting mechanical energy. Adv. Mater. 2014, 26, 3580.

(19) Jeong, S. M.; Song, S.; Lee, S.-K.; Ha, N. Y. Color manipulation of mechanoluminescence from stress-activated composite films. Adv. Mater. 2013, 25, 6194.

(20) Jeong, S. M.; Song, S.; Joo, K.-I.; Kim, J.; Hwang, S.-H.; Jeong, J.; Kim, H. Bright, wind-driven white mechanoluminescence from zinc sulphide microparticles embedded in a polydimethylsiloxane elastomer. Energy Environ. Sci. 2014, 7, 3338.

(21) Wong, M.-C.; Chen, L.; Bai, G.; Huang, L. B.; Hao, J. Temporal and Remote Tuning of Piezophotonic-Effect-Induced Luminescence and Color Gamut via Modulating Magnetic Field. Adv. Mater. 2017, 29, 1701945.

(22) Qian, X.; Cai, Z.; Su, M.; Li, F.; Fang, W.; Li, Y.; Zhou, X.; Li, Q.; Feng, X.; Li, W.; Hu, X.; Wang, X.; Pan, C.; Song, Y. Printable Skin-Driven Mechanoluminescence Devices via Nanodoped Matrix Modification. Adv. Mater. 2018, 30, 1800291.

(23) Smet, P. F.; Moreels, I.; Hens, Z.; Poelman, D. Luminescence in Sulfides: A Rich History and a Bright Future. Materials 2010, 3, 2834.

(24) Wang, J.; Yan, C.; Cai, G.; Cui, M.; Lee-Sie Eh, A.; See Lee, P. Extremely Stretchable Electroluminescent Devices with Ionic Conductors. Adv. Mater. 2016, 28, 4490.

(25) Xu, X.; Hu, D.; Yan, L.; Fang, S.; Shen, C.; Loo, Y.-L.; Lin, Y.; Haines, C. S.; Li, N.; Zakhidov, A. A.; Meng, H.; Baughman, R. H.; Huang, W. Polar-Electrode-Bridged Electroluminescent Displays: 2D Sensors Remotely Communicating Optically. Adv. Mater. 2017, 29, 1703552.

(26) Peng, W. Q.; Cong, G. W.; Qu, S. C.; Wang, Z. G. Synthesis and photoluminescence of $\mathrm{ZnS}: \mathrm{Cu}$ nanoparticles. Opt. Mater. 2006, 29, 313.

(27) Chandra, B. P.; Chandra, V. K.; Jha, P. Piezoelectricallyinduced trap-depth reduction model of elastico-mechanoluminescent materials. Phys. B 2015, 461, 38.

(28) Jeong, S. M.; Song, S.; Lee, S.-K.; Choi, B. Mechanically driven light-generator with high durability. Appl. Phys. Lett. 2013, 102, 051110 .

(29) Wang, F.; Wang, F.; Wang, X.; Wang, S.; Jiang, J.; Liu, Q.; Hao, X.; Han, L.; Wang, J.; Pan, C.; Liu, H.; Sang, Y. Mechanoluminescence enhancement of $\mathrm{ZnS}: \mathrm{Cu}, \mathrm{Mn}$ with piezotronic effect induced trap-depth reduction originated from PVDF ferroelectric film. Nano Energy 2019, 63, 103861.

(30) Bai, P.; Zhu, G.; Lin, Z.-H.; Jing, Q.; Chen, J.; Zhang, G.; Ma, J.; Wang, Z. L. Integrated multilayered triboelectric nanogenerator for harvesting biomechanical energy from human motions. ACS Nano 2013, 7, 3713.

(31) Zhou, Y. S.; Liu, Y.; Zhu, G.; Lin, Z.-H.; Pan, C.; Jing, Q.; Wang, Z. L. In situ quantitative study of nanoscale triboelectrification and patterning. Nano Lett. 2013, 13, 2771.

(32) Wang, S.; Lin, L.; Xie, Y.; Jing, Q.; Niu, S.; Wang, Z. L. Slidingtriboelectric nanogenerators based on in-plane charge-separation mechanism. Nano Lett. 2013, 13, 2226.

(33) Pearson, R. A.; Yee, A. F. Influence of particle size and particle size distribution on toughening mechanisms in rubber-modified epoxies. J. Mater. Sci. 1991, 26, 3828.

(34) Zou, H.; Zhang, Y.; Guo, L.; Wang, P.; He, X.; Dai, G.; Zheng, H.; Chen, C.; Wang, A. C.; Xu, C.; Wang, Z. L. Quantifying the triboelectric series. Nat. Commun. 2019, 10, 1427.

(35) Wei, X. Y.; Zhu, G.; Wang, Z. L. Surface-charge engineering for high-performance triboelectric nanogenerator based on identical electrification materials. Nano Energy 2014, 10, 83. 